phys. stat. sol.

$-1-$

\title{
Quantum and thermal effects in the double exchange fer- romagnet
}

\author{
Nic Shannon \\ Max-Planck-Institut für Physik komplexer Systeme, Nöthnitzer Str. 38, 01187 \\ Dresden, Germany \\ (Submitted — Physica Status Solidi (b) 236, 494 (2003)) \\ Subject classification: $75.25 .+\mathrm{z}$
}

\begin{abstract}
The physics of the ferromagnetic phase of the "double exchange" model has been widely discussed in the context of the CMR manganites. Usually, the double exchange ferromagnet is treated is classically, by mapping it onto an effective Heisenberg model. However this mapping does not permit a correct treatment of quantum or thermal fluctuation effects, and the results obtained lack many of the interesting features seen in experiments on the manganites. Here we outline a new analytic approach to systematically evaluating quantum and thermal corrections to the magnetic and electronic properties of the double exchange ferromagnet.
\end{abstract}

Ferromagnetic manganites and double exchange Systems in which charge carriers move in a background of local magnetic moments are ubiquitous among doped $\mathrm{d}$-electron oxides, the two most widely studied examples being the colossal magnetoresistance (CMR) manganites and the high temperature superconducting (HTc) cuprates.

It was first suggested by Zener [1] that ferromagnetism in the manganites originated from the kinetic energy of the doped holes, which couple to the local moments of the Mn atoms through a strong Hund's first rule coupling. This idea was later formalised in terms of the strong coupling limit of a ferromagnetic Kondo lattice Hamiltonian [2, 3]

$$
\mathcal{H}=-t \sum_{\langle i j\rangle \alpha} c_{i \alpha}^{\dagger} c_{j \alpha}-J \sum_{i} \vec{S}_{i} \cdot \vec{s}_{i} \quad \vec{s}_{i}=\frac{1}{2} \sum_{\alpha \beta} c_{i \alpha}^{\dagger} \sigma_{\alpha \beta} c_{i \beta}
$$

Here, $t$ is the hopping integral for $e_{g}$ electrons transfered between neighbouring $\mathrm{Mn}$ atoms, whose half filled $t_{2 g}$ levels are modelled as a spin $S=3 / 2$ local moment, and $J \gg t>0$ is the Hund's rule coupling between itinerant and localised electrons. The orbital degeneracy of the $e_{g}$ levels (which are Jahn-Teller active) has been neglected, as have next nearest neighbour hoppings and all Heisenberg super-exchange processes. This minimal model - a single band of tight binding electrons coupled to a background of local moments is for historical reasons known as the "double exchange" (DE) model, and we will refer to its Ferromagnetic ground state as the "double exchange ferromagnet" (DEFM).

The model Equation (1) has been widely studied for fifty years, and we shall not pretend here to offer a review of either its diverse physical properties, or of the many ingenious approaches which have been used to study them. Rather, we present a concise introduction to a simple and controlled way of performing a spin wave expansion for such a Hamiltonian, and a brief overview of the effect of quantum and thermal fluctuations on the DEFM. We believe that the methods developed can easily be transferred to other magnetic d-electron oxides. 
a)


b)

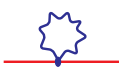


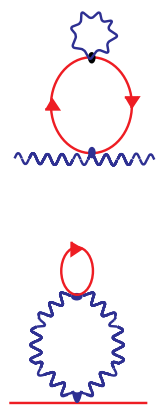

Figure 1: Diagrams contributing to the self energy of a) spin waves and b) low energy spin-projected electrons to order $\mathcal{O}\left(1 / S^{2}\right)$.

Spin wave Expansion In the strong Hund's rule coupling limit $t / J \rightarrow 0$, so far as magnetic properties are concerned, it makes little sense to distinguish between localised and itinerant electrons. For example, oxidised $\mathrm{Mn}$ atoms in the canonical CMR manganite $\mathrm{La}_{1-x} \mathrm{Ca}_{x} \mathrm{MnO}_{3}$ can exist either as $\mathrm{Mn}^{4+}$ of $\mathrm{Mn}^{3+}$ ions, which are respectively spin $\mathrm{S}=3 / 2$ and spin $\mathrm{S}=2$ local moments. Where the Hund's rule coupling is so strong, it is therefore convenient to work in the local basis of eigenstates of total (i.e. "itinerant" $e_{g}$ plus "localised" $t_{2 g}$ electron) spin $\vec{T}=\vec{S}+\vec{s}$.

Taking this as a starting point, we have derived a controlled large $S$ expansion of Equation (1) which can be applied to any magnetically ordered state. Our original approach $[5,6,7,8]$ was to generalise the Holstein-Primakoff (HP) transformation [4] by allowing the length of the fluctuating spin $\mathrm{T}$ to be an operator quantity, and introducing local "up" $(f)$ and local "down" $(p)$ electron operators such that $T=S+\left(f^{\dagger} f-p^{\dagger} p\right) / 2$. A more general derivation of the associated spin and charge algebra based on the Schwinger Boson representation of Penc and Lacaze [9] will be presented elsewhere [10].

Where the operator $f^{\dagger}$ acts on an empty site, it creates a low energy state with total spin $T=S+1 / 2$, while the the operator $p^{\dagger}$ creates a high energy state with total spin $T=S-1 / 2$. All information about low energy spin excitations is encoded in the generalised Holstein-Primakoff bosons $\tilde{a}$, so in the limit $t / J \rightarrow 0$, we can project out the high energy, low spin states associated with $p$ electrons altogether, and obtain a very simple representation of Equation (1) :

$$
\begin{aligned}
\mathcal{H}^{\prime}= & \sum_{k_{1}} \epsilon_{1} f_{1}^{\dagger} f_{1}+\frac{1}{N} \sum_{k_{1} \ldots k_{4}} v_{24}^{13} f_{1}^{\dagger} f_{2} \tilde{a}_{3}^{\dagger} \tilde{a}_{4} \delta_{1+3-2-4} \\
& v_{24}^{13}=\frac{1}{4\left(S+\frac{1}{2}\right)}\left[\left(1+\frac{1}{8 S}\right)\left(\epsilon_{1+3}+\epsilon_{2+4}\right)-\left(\epsilon_{1}+\epsilon_{2}\right)\right]
\end{aligned}
$$

where $\epsilon_{k}$ is the (tight binding) electron dispersion and $v_{24}^{13}$ the vertex for interaction between band electron $(f)$ and spin wave modes $(\tilde{a})$. For compactness, a further four-boson/twofermion vertex has been dropped. The generalisation of this Hamiltonian to bilayer systems is straightforward [11].

From this starting point it is possible to use a conventional diagrammatic perturbation theory to evaluate spin-wave and electron self energy corrections. The leading zero temperature semi-classical process is the one loop diagram of Figure (1a), which generates a dispersion for the spin waves, and so stabilises the FM ground state. 
phys. stat. sol.

Quantum and Thermal Effects At a semi-classical level (i.e. considering only the $\mathcal{O}(1 / S)$ one loop diagrams of Figure (1a and $1 \mathrm{~b})$ ) the DEFM can be mapped onto an independent set of spin excitations, described by a nearest-neighbour Heisenberg model (see e. g. $[3,7]$ ), and a band of tight binding electrons with temperature dependant bandwidth $[12]$.

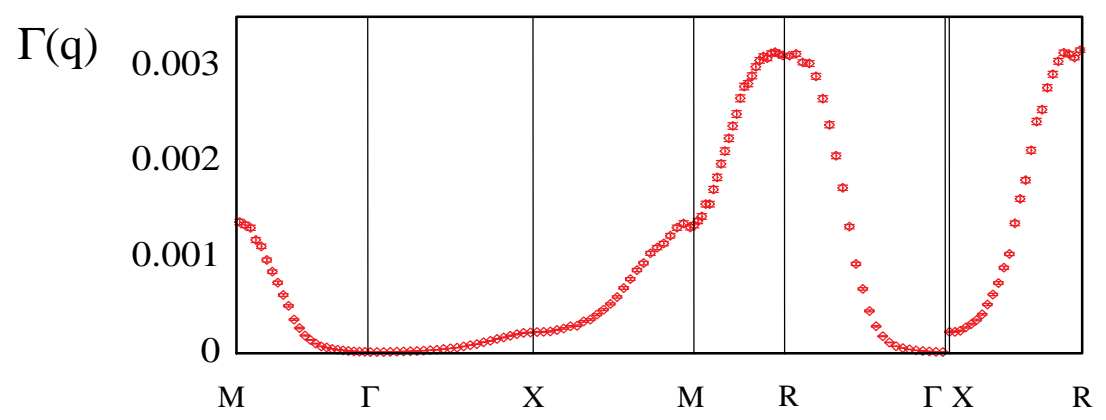

Figure 2: Damping of spin waves in a DEFM with electron doping $\mathrm{x}=0.7$, at zero temperature, throughout the Brillouin zone. Energies are measured in units of the electron bandwidth $2 z t=1.0$.

Once the leading quantum effects are taken into account (the remaining $\mathcal{O}\left(1 / S^{2}\right)$ diagrams of Figure (1)), this mapping breaks down. The spin waves become damped (see Figure (2)), even at zero temperature, and both quantum and thermal fluctuations dynamically generate new non-nearest neighbour couplings between spins, which progressively modify the form of spin wave dispersion (see Figure (3)).

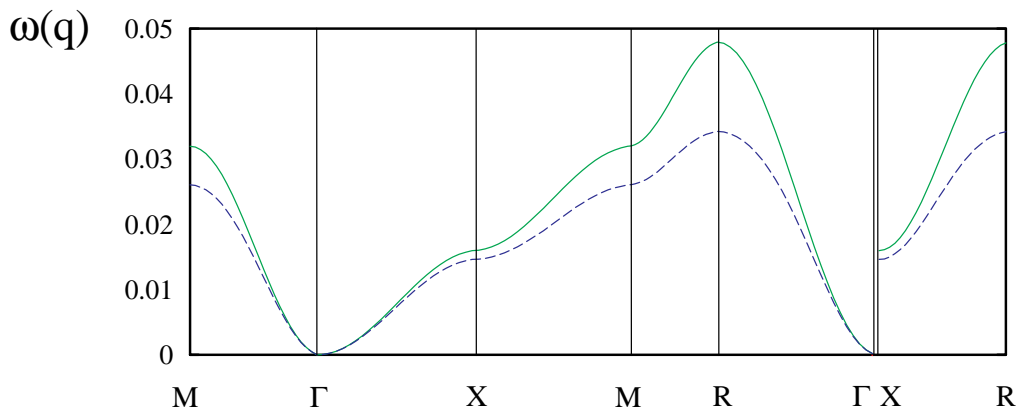

Figure 3: Spin wave spectrum of the DEFM with electron doping $\mathrm{x}=0.7$ at zero temperature. Upper line - semi-classical result. Lower line - result when leading quantum corrections are taken into account. Energies are measured in units of the electron bandwidth $2 z t=1.0$.

Thermal corrections to the overall scale of the spin wave dispersion in the DEFM are broadly similar to those in the Heisenberg FM, but are considerably enhanced relative to the classically equivalent Heisenberg model (see Figure (4)). 
At zero temperature, in the limit $t / J \rightarrow 0$, the low energy $f$ electron states remain undamped. However at finite temperature they interact through the transverse spin susceptibility, and have a finite lifetime which is controlled by the phase space for spin fluctuations $[12]$.

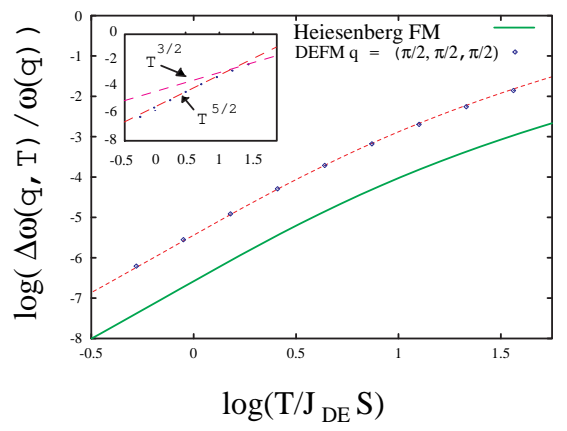

Figure 4: Log-log plot of finite temperature corrections to the spin wave spectrum of the DEFM, together with those of the semi-classically equivalent Heisenberg FM. Temperature corrections $\Delta \omega(q, T)$ are normalised to bare dispersion $\omega(q)$, temperatures to the scale of spin wave dispersion $J_{D E} S$.

Conclusions We have shown that it is possible to derive a controlled large $S$ expansion for the Kondo lattice which, when applied to the DEFM in the limit $t / J \rightarrow 0$, reduces the model to a set of composite spin modes interacting with spinless electrons. At a semi-classical level spin and charge modes do not interact, but at $\mathcal{O}\left(1 / S^{2}\right)$ interactions are restored and the physics of the system substantially modified.

At a qualitative level, the departures from Heisenberg model behaviour caused by quantum and thermal fluctuation effects in the DEFM resemble those seen in inelastic neutron scattering experiments on the CMR manganites. However there remains some doubt that a minimal model of the form Equation (1) is sufficient to fit experimental data for the manganites quantitatively [11].

\section{References}

[1] C. Zener, Phys. Rev., 82, 403 (1951).

[2] P. W. Anderson, and H. Hasegawa, Phys. Rev., 100, 675 (1955).

[3] P. G. de Gennes, Phys. Rev., 118, 141-154 (1960).

[4] Holstein and Primakoff, Phys. Rev., 58, 1098-1113 (1940).

[5] N. Shannon and A. V. Chubukov, cond-mat/0011390 - J. Phys.: Condens. Matter, 14, L235241 (2002).

[6] N. Shannon, J. Phys.: Condens. Matter, 13, 6371-6386 (2001).

[7] N. Shannon, and A. Chubukov, Phys. Rev. B, 65, 104418 (2002).

[8] N. Shannon, to appear in proceedings of VI Vietri training course in Physics of Correlated Electron Systems and Superconductivity, cond-mat/0203156.

[9] K. Penc and R. Lacaze, Europhys. Lett. 48, 561 (1999).

[10] N. Shannon and K. Penc, in preparation.

[11] N. Shannon, T. Chatterji, F. Ouchini, and P. Thalmeier. Eur. Phys. J. B, 27, 287 (2002).

[12] N. Shannon, unpublished. 